\title{
Nanotechnology a Modern Arsenal in Crop Disease Management
}

\section{Taswar Ahsan*}

Department of Plant Pathology, College of Plant Protection, Shenyang Agricultural University, Shenyang, Liaoning, China

*Corresponding Author: Taswar Ahsan, Department of Plant Pathology, College of Plant Protection, Shenyang Agricultural University, Shenyang, Liaoning, China.

Received: September 23, 2019; Published: September 30, 2019

As the population increased day by day through out the world. The demand of the food will be doubled by 2050 . Crops are continues facing the pathogens including virus, bacteria, fungi and insects. Every year faced heavy economic loss. To manage the plant disease there are several methods including chemical based compounds, biocontrol agents, transgenic plants and genetically modified plants. Chemical methods have harmful effects for human health and as well as for biodiversity. Several pathogens adopt resistance against biocontrol agents and transgenic plants facing tough time from the pathogens. For crop disease management there is emergence need to adopt novel efficient approaches. Now a days nanotechnology is an efficient practice for plant protection. The application of nanotechnology comprises of plant or microbes extract with metals and formed nanoparticles. Any material that has one or more dimensions at the scale of 1 to $100 \mathrm{~nm}$ are termed as nanoparticles. Synthesized nanoparticles can be produced to specific dimensions and designed in countless composite arrays, making their function and utility applicable within many disciplines. Nanoparticles can be synthesized by various protocols. Several nanoparticles utilized in human health management, while presently there are still need exploration in crop disease management. Many of existed nanoparticle could be used in crop protection management. Now a days nanoparticles of metalloids, metallic oxides, nonmetals (single and composites), carbon nanomaterials (single- and multiwalled carbon nanotubes, graphene oxides, and fullerenes), and functionalized forms of dendrimers, liposomes, and quantum dots have begun to infiltrate plant pathology. Nanotechnology will enable the development of multiple new methods for suppressing disease in the greenhouse and field, enhance disease diagnostics, and create many new tools for molecular manipulations of plants and pathogens. Nanoparticles have several applications in crop disease management, such as disease diagnose, antimicrobial drug, drug delivery and nanofertilizer. In future nanotechnology will have big role in food industry. It could be sustainable and environment friendly approach. Especially in crop disease management nanotechnology would be major impact.

\section{Volume 3 Issue 10 October 2019 (C) All rights are reserved by Taswar Ahsan.}

